\title{
Evaluating New Interaction Paradigms in SEN Teaching: Defining the Experiment
}

\author{
Paloma Cantón ${ }^{1}$, José L. Fuertes ${ }^{2}$, Ángel L. González ${ }^{2}$ and Loïc Martínez ${ }^{2}$ \\ ${ }^{1}$ Consejería de Educación de la Comunidad de Madrid, Spain \\ pcantoneeduca.madrid.org \\ ${ }^{2}$ School of Computer Science and Engineering, Technical University of Madrid, \\ Boadilla del Monte, Madrid, Spain \\ \{jfuertes, agonzalez, loic\}@fi.upm.es
}

\begin{abstract}
New devices have made their way into everyday life in recent years, opening the doors to new ways of interacting with computers, providing different, and potentially better, solutions to some problems. But this raises the question of if there is any way of measuring whether or not these new devices are suitable. This paper presents a strategy for evaluating the suitability of new interaction devices in the context of teaching children with special educational needs.
\end{abstract}

\section{Introduction}

New devices have made their way into everyday life in recent years, opening the doors to new ways of interacting with computers. This affords the opportunity to devise new systems to exploit these new interaction paradigms and provide different, and potentially better, solutions to some problems, like the teaching of children with special educational needs (SEN).

The question raised is whether it is a good idea to use these devices in teaching systems targeting SEN users and whether there is any way of measuring the suitability of these new paradigms. Furthermore, new metaphors of interaction associated with each new device will have to be defined, and it will be necessary to validate whether or not each metaphor is suitable or needs to be tailored.

Therefore, we believe that a protocol should be established to measure and compare how usable an interaction device is and how the user would interact with the system using the respective device in a SEN context.

This paper outlines the first step towards this objective, which involves:

- selecting the user types who will participate in the tests and the device types to be compared

- developing a system that will act as a testbench for the devices 
- defining the information to be gathered both automatically by the system and manually by an observer

- defining the experimental procedure.

In the following we will explain the state of the art, the methodology applied to define the experiment and the conclusions and future lines of research.

\section{Related Work}

In recent years, many newly designed devices have changed the face of humancomputer interaction. Some examples are games systems (Wii, DS), mobile devices (tablets or phones) or other devices (tactile screens and Kinect). In this section, we will look at some approaches in this field and their educational applications.

Seven years ago Apple revolutionized the field of mobile devices by providing support for user interaction via gestures and actions. Apple introduced two concepts: touches and gestures. Google built a similar touch- and gesture-based system into its Android operating system for mobile devices. Microsoft has developed a similar effort for interacting with Microsoft software using gestures (from Windows 8 to Kinect) [1].

There have been different initiatives to use these kinds of new interaction devices to improve education. Technology and education can be combined at all educational levels, but a conceptual framework should be used that identifies the required knowledge (content, teaching method, and technology) and supports teachers with technology integration [2].

In this context, children usually show clear preferences for games using relatively simpler interaction modes; they mostly prefer to play touch games (using a multitouchable display) than computer games (using mouse and keyboard) [3].

Another interesting learning approach is based on a collaborative game played by six players with handheld motion-sensitive tangible user interfaces in front of a large shared display. The game teaches children how to cooperate with each other (social skills) and collectively reason about the cause-and-effect relationships of the different mechanisms required to move, saw and lift planks (problem-solving skills) [4].

Another type of device enables touchless gestures, that is, gestures performed in the air without touching a device. One example is Microsoft Kinect, a new device for the Xbox 360 game system launched in 2010 (which can also be used on Windows computers) [5]. Kinect set out to change the way gamers usually play videogames by converting their body into the "game controller" controlling the different elements of the game and interacting through a natural user interface using gestures and spoken commands.

Kinect was initially released as a gaming device, but it has been used as an artificial vision device in many areas of application by researchers worldwide. Research into Kinect is growing, but, so far, there is not much literature on the adaptation of this technology for children and young people with disabilities. Nevertheless, Kinect had been used effectively by people with special needs and as an auxiliary learning tool in the traditional classroom. Some successful experiences with Kinect are: improvement of the outcomes of a daily life game used by children with autism, a system of drawing via hand motion, a system to create music by arm motion [6], a system to 
help immigrant children learn a new language for the purposes of classroom learning and integration into local society [7], or a system for rehabbing students' motor skills, enhancing the memory of people with autism and encouraging greater sociability (helping them to cooperate with each other and gradually develop their oral expression to a point where they can give basic instructions to each other during the game) [8]. An interactive framework using Kinect for e-learning specific for presentation and assessment has also been published [9]. The goal is to create a virtual smart classroom environment rendering the classroom more interactive and exciting.

To sum up, touch and touchless gesture systems are able to enhance classroom interactions, increase participation, improve teachers' ability to present and manipulate multimodal materials, create opportunities for interaction and discussion, kindle student creativity, afford technical interactivity (including children with SEN), enhance teaching, or support learning. But as researchers are not yet clear about how the application of gesture-based computing improves learning, these devices need empirical evidence to support their legitimacy as an adequate educational technology [10] and to identify their suitability for different situations and educational environments.

\section{$3 \quad$ Methodology}

The process followed to develop the research was composed of the following steps:

- Identify the profile of participant users.

- Select the devices to be tested as part of this first interaction.

- Develop an educational game to test and compare devices.

- Define the information to be gathered by the system and the educator accompanying the child during the experiment (to be performed at the same time as the last step).

- Define the experiment protocol to assure reproducible and consistent results.

\subsection{Identifying the Profile of Participant Users}

The SEN users that most often use and find it easier to access these ICT devices have mild intellectual disability. On this ground, our study commences with this user population. This user group does not have any real trouble handling the target devices, although they do not use them as precisely, rapidly and skilfully as other users generally do. The study sets out to answer the following questions: What problems do they have? How do they show up? What influence does their intellectual disability have on the use of the selected devices?

\subsection{Selecting Devices}

At the start of this research project in September 2012 we found that the interaction devices that the educational field was starting to try out were mostly touch-sensitive 
devices and contactless gesture interaction devices. The first group includes mobile telephones, tablets and touchscreen computers. The second group includes webcams, motion sensors derived from the technology developed by PrimeSense [10] and other promising sensors like Leap Motion [11].

As the aim is to be able to develop a system to compare different types of devices, they logically had to be tested in the same environment. On this ground, we opted to develop the system on a personal computer running Windows 7 or Windows 8 . The selected devices were a multi-touch screen and Microsoft Kinect.

\subsection{Developing the Educational Game}

We have designed an educational game that covers the most common movements used in the selected devices in order to analyse how they are performed by users and how user characteristics influence their performance.

The subject matter and operation of the game was defined in partnership with a teaching specialist, and activities in which the above movements are suitably functional and coherent were selected. The characteristics of the target users was another factor taken into account in the design of the activity, which was tailored to their educational level, the teaching goals for the user group and their gaming preferences. While the game has to be attractive, dynamic and interactive enough to motivate the users to play, it must not contain so many stimuli as to distract users and render it overly complex. Thus, the graphical interface of the games was designed to be simple and attractive.

Defining the Games. The best practice for defining the games that were to be part of the experiment was considered to be to use a subject matter that had the potential to test and compare several gestures using the selected devices. During this phase, we concluded that the actions that the user would have to perform to complete the game should include vertical, horizontal and wrist-twisting movements. We also decided that the child should be allowed to use non-educational games to learn how to operate the devices they are to use.

The first of these training activities is a bowling game, including skittles and coloured bowling balls. The player has to select and roll the respective coloured bowling ball. In the second training activity, the user has to use a set of letters to form a word. This game uses the same actions as the bowling game, plus the open action. In the same vein of gradually adding new actions to the activities, we designed a classification game organized at three difficulty levels (Fig. 1). At the easy level, the user has to select the respective element at the top of the screen (cloud), drop it on to the belt and then knock it into a round box. At the normal level, the stop box rotation and open box actions are added. At the hard level, the rotate gesture is added to the above. 


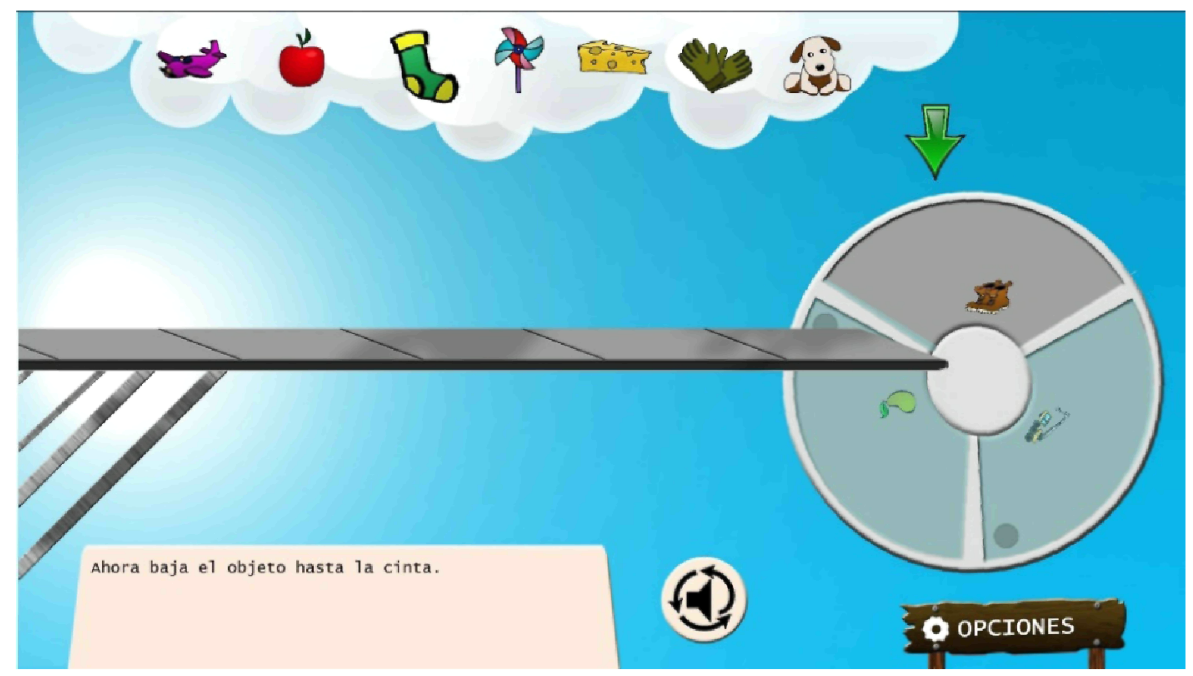

Fig. 1. Classification game

Defining the Interaction Vocabulary. The gestures denoting the actions that the user is to perform vary depending on the selected device. We focus on the gestures that are most commonly used in activities targeting younger users, which tend to be the most intuitive and look, on paper, to be the simplest to perform.

The gestures have been defined taking into account that the aim is to rate particular skills or abilities when moving certain body parts or performing hand gestures:

- Sweeping movements of arms, like up-down, sideward, in-out, rotational motions, etc.

- One-/two-handed movements: simple motions like touching part of the screen or moving right/left or up/down on the screen.

- One-finger movements: simple motions like touching or moving around the screen.

- Two-finger movements: complex motions like pinching with the index finger and thumb.

Additionally, these movements must be performed at a particular speed, which may vary. Faster movements are harder to execute properly than slower ones. These movements can be used to interact with the device applications and perform actions like select, execute, move/drag, zoom, etc.

By examining these movements and how effectively they are performed by users with an intellectual disability, we will be able to analyse what requirements the new interaction paradigms introduce and what competences users require for their performance. We believe that these gestures and body movements may be a limiting factor for users with intellectual difficulties, because, although they do not have physical handicaps, they do have problems with hand-eye coordination, precision, speed or visual-spatial processing, which can have a bearing on the effectiveness with which they perform these gestures to solve the assigned activities.

Fig. 2 shows the gesture defined for knocking an object into a box using Kinect and Fig. 3 illustrates the equivalent gesture for a tactile screen. 

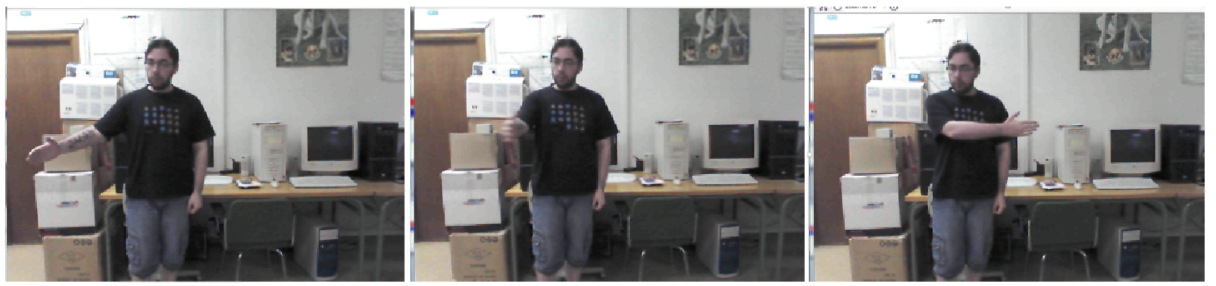

Fig. 2. Kinect gesture for knocking objects into the box

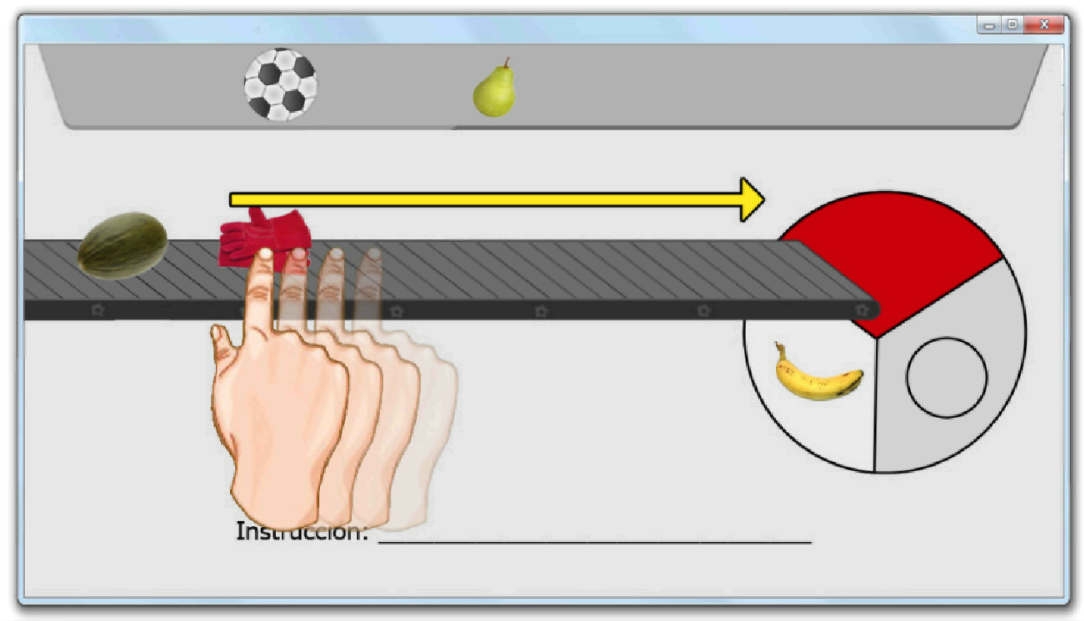

Fig. 3. Touchscreen gestures for knocking objects into the box

\subsection{Defining the Information to be Gathered}

There are two sources of information: information gathered automatically by the game and information gathered by the teacher after users have completed the activity.

The game is able to automatically collect a variety of generic (successes, failures...) and device-specific data. For touchscreen actions it measures gesture precision. To do this, a region is defined around the touch path trajectory. If the gesture runs through the centre of this region, precision is rated as $100 \%$. The precision decreases as the trajectory moves away from the centre. The precision is $0 \%$ if the gesture is outside the region. This region is not visible to users. For Kinect, data are collected on how often the gesture is performed incorrectly before it is performed correctly.

In summary, the captured data will measure the number of successes, failures, precision and time taken to perform the gaming actions. From these data, we will be able to draw conclusions about the manipulative skills (precision, speed), efficiency and effectiveness of the users when performing the task and how the use of these devices influences the attainment of teaching goals. 
On the other hand, the teacher completes a questionnaire designed to gather additional information regarding teaching and system user experience:

- Session data, like session time and whether or not the child had used the system before

- User personal data: any learning, visual and motor disabilities, as well as age and sex

- Data on gaming performance, recording observed gestures, postures and mobility problems

- Questions on user experience based on standard usability questionnaires.

\subsection{Experimental Process}

We have established a four-phase process in order to develop the experiment. Depending on the results of each phase, it may be necessary to revise the defined process and tailor the experiment in order to achieve more reliable results.

The phases are:

1. Functionality test: evaluate the system with 6- to 11-year-old children without SEN at our facilities. The goal is to check that the game works properly with respect to both activity performance and data collection. The questionnaire will not be used to collect data at this stage.

2. Pilot test: evaluate the system in a real environment (at a school) with 10 to 12 children aged from 6 to 11 years without SEN. The teacher monitoring the activity will take the survey after each child finishes using the system. This will serve to check that the system works in a real-world situation and that the questionnaire is adequate for collecting additional data.

3. Experiment: visit several schools that have pupils with mild intellectual disability. The pupils will perform the set activities, the system will collect data and the teacher will complete the respective questionnaires in accordance with the experimental protocol refined in phase 2 .

4. Results analysis: the results of phase 3 will be analysed in order to determine and compare the adequacy of the multipoint touchscreens and the Kinect system for educational systems to be used by children with special educational needs.

Phase 1 was performed in February 2014 and the initial results show that the children were able to perform the tasks without too much trouble, but the gesture recognition system needed some refinement to increase its reliability. Once the system has been upgraded we will enact phases 2 and 3 from October to December 2014. If all goes according to plan, phase 4 will get under way in February 2015.

\section{Conclusions and Future Lines of Research}

New devices and ways of interacting with machines have emerged in recent years. But there is no standardized means of assessing which of these devices to use and how to use them in each context. 
This paper reports the design of an experiment and the development of an educational application whose architecture can straightforwardly accommodate new devices and interaction paradigms. In this manner, it will be easy to compare the adequacy of each device in a particular context.

\section{References}

1. Cantón, P., González, Á.L., Mariscal, G., Ruiz, C.: Applying New Interaction Paradigms to the Education of Children with Special Educational Needs. In: Miesenberger, K., Karshmer, A., Penaz, P., Zagler, W. (eds.) ICCHP 2012, Part I. LNCS, vol. 7382, pp. 65-72. Springer, Heidelberg (2012)

2. Tse, E., Marentette, L., Ahmed, S.I., Thayer, A., Huber, J., Mühlhäuser, M., Kim, S.J., Brown, Q.: Educational Interfaces, Software, and Technology. In: Proc. CHI 2012, pp. 2691-2694. ACM (2012)

3. Yu, X., Zhang, M., Ren, J., Zhao, H., Zhu, Z.: Experimental Development of Competitive Digital Educational Games on Multi-touch Screen for Young Children. In: Zhang, X., Zhong, S., Pan, Z., Wong, K., Yun, R. (eds.) Edutainment 2010. LNCS, vol.6249, pp. 367-375. Springer, Heidelberg (2010)

4. Goh, W.-B., Fitriani, G.C.-F., Menon, M., Tan, J., Cohen, L.G.: Action Role Design and Observations in a Gestural Interface-based Collaborative Game. In: Proc. CHI 2011, pp. 763-772. ACM (2011)

5. Zhang, Z.: Microsoft Kinect Sensor and Its Effect. IEEE Multimedia 19(2), 4-10 (2012)

6. Bossavit, B., Pina, A.: An interdisciplinary methodology for designing and implementing educational tools for children and youth with special needs. SIGAccess Newsletter 105, 4-8 (2013)

7. Mateu, J., Lasala, M.J., Alamán, X.: Tangible Interfaces and Virtual Worlds: A New Environment for Inclusive Education. In: Urzaiz, G., Ochoa, S.F., Bravo, J., Chen, L.L., Oliveira, J. (eds.) UCAmI 2013. LNCS, vol. 8276, pp. 119-126. Springer, Heidelberg (2013)

8. Boutsika, E.: Kinect in Education: A Proposal for Children with Autism. In: Proc. DSAI 2013. Elsevier (2013)

9. Sommool, W., Battulga, B., Shih, T.K., Hwang, W.-Y.: Using Kinect for Holodeck Classroom: A Framework for Presentation and Assessment. In: Wang, J.-F., Lau, R. (eds.) ICWL 2013. LNCS, vol. 8167, pp. 40-49. Springer, Heidelberg (2013)

10. Hsu, H.J.: The Potential of Kinect in Education. International Journal of Information and Education Technology 1(5), 365-370 (2011)

11. PrimeSense. 3D Sensing Technology Solutions (2014), http : / /www primesense.com/

12. Leap Motion. Mac \& PC Motion Controller for Games, Design and More (2014), https : / / www. leapmotion.com/ 